\title{
APPLICATION OF A BP NEURAL NETWORK IN ELECTRICAL AUTOMATIONS
}

\author{
Zhenhua Han \\ School of Electronic and Electrical Engineering, Zibo Vocational Institute, Zibo, 255000, China. \\ Email: hanzhenhua55@163.com
}

\begin{abstract}
In order to further achieve the goal of automation in the field of electrical engineering, with the short-term load forecasting of power system as the research object, the artificial neural network algorithm- BP (Back-Propagation) algorithm and optimization, the establishment of impact factor mapping database and other related theories are used. Based on the theoretical results of many researchers in China and foreign countries, a data model based on BP neural network is established and optimized by genetic algorithm; then, the mapping database of factors affecting short-term load of power system is established, which are divided into three categories: working day type, meteorological factors and temperature factors. The importance of these three factors is reflected by the value of human mapping; in order to avoid the over fitting of data, the initial sample data needs to be normalized. Then, with the historical data of the power load in Songjiang District in Shanghai as a sample, the neural network is trained and simulated. Finally, the error between the prediction results and the real data is compared. The results show that the trained neural network technology has high accuracy and reasonable error and average deviation for short-term power load forecasting. Meanwhile, after comparison, when meteorological factors and working day factors are considered, the prediction error is obviously less than that without considering both. It shows that the trained relevant factors can improve the accuracy of the prediction after quantitative mapping. Moreover, the more complete the database of influence factor mapping is, the higher the accuracy of load forecasting by using neural network is. Thus, BP neural network plays a more important role in the field of electrical automation.
\end{abstract}

Keywords: Neural Network; Power System; Automation; Mapping Database; Genetic Algorithm.

\section{Introduction}

In the process of building a powerful socialist country, electrical engineering and its automation, as the key field of industrial development, has been the concern of scientific researchers from all walks of life. With the continuous progress of science and technology in recent years, as well as the continuous development of China's economy, great progress has been made in social development, industrial system, scientific research technology and other aspects [1]. As a society dominated by industry, only by vigorously developing industry can China promote the rapid development of economy [2]. Throughout the development of China's industrial economy, electrical automation engineering plays a considerable role in it. Among them, the electrical automation engineering control system helps enterprises reduce economic costs, and improve the detection accuracy $[3,4]$. With the development of intelligent simulation, electronic technology and computer network, electrical automation technology develops rapidly. It not only becomes a comprehensive technology that integrates the advantages of all parties, but also improves the stability of the whole system while realizing electrical automatic control [5]. However, although China has achieved remarkable results in the development of electrical automation, compared with some developed countries in the world, there are still obvious shortcomings, mainly in the digital and innovative application of electrical automation control system [6].

Nowadays, the world has entered the era of digital information, and all walks of life are gradually realizing modernization reform with the help of the trend of digital. For the field of electrical automation, it is more important to realize digital information, so digitization is the trend of the development of electrical engineering automation system in the future [7]. The data of the whole information is input into the computer as the target, processed and classified by algorithm, and finally output as required, in order to gradually realize the 
digitalization of the electrical automation engineering control system construction [8]. As a new data processing model, neural network can not only stack multiple algorithms to achieve the functions of data identification, classification and processing, but also can continuously optimize the algorithm to improve efficiency, and apply it to many different fields to realize the transformation and upgrading of digital and information [9]. The establishment of BP (Back-Propagation) neural network digital model to predict the power load is a milestone in the field of electrical engineering to realize automation [10]. BP neural network is used to build a digital model, genetic optimization algorithm is used to optimize the neural network by training, and then perturbation algorithm is used to enhance the accuracy of prediction results. Finally, the perfect power load forecasting system is established, which provides a feasible scheme for the realization of digital operation in the electrical field.

\section{BP Neural Network Algorithm and Data Optimization 2.1 The Concept of Neural Network}

Artificial neural network is composed of several neural units. It is a mathematical model processor with parallel functions of learning, storing knowledge, classifying, processing and judging data [11]. Figure 1 is a model of the neural unit.

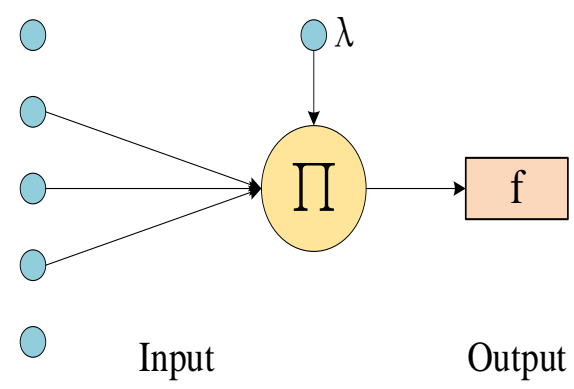

Figure 1: BP neural network model

According to its network structure, it can be divided into three categories: single layer feedforward neural network, multilayer feedforward neural network and recurrent neural network [12]. Among them, single layer feedforward neural network refers to the arrangement of neurons by layer, which respectively constitute input layer and output layer, without hidden layer; signal enters the output layer from input layer and then directly outputs. The single layer feedforward neural network is simple in structure, each neuron can only accept input from the previous layer of neurons, and the output layer has no signal feedback to the input layer. Figure 2 presents its structural model.

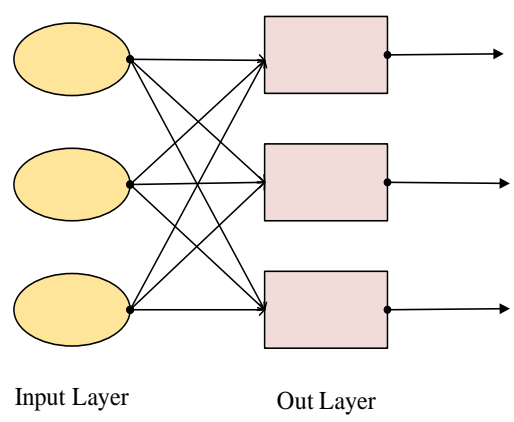

Figure 2: Single layer feedforward neural network structure

Compared with the single layer feedforward neural network, the hidden layer is added in the multilayer feedforward neural network. Its function is to use a specific algorithm to adjust the relationship between the input layer and the output layer signal, so as to get the data that meets the requirements and finally out. Figure 3 is the structural model.

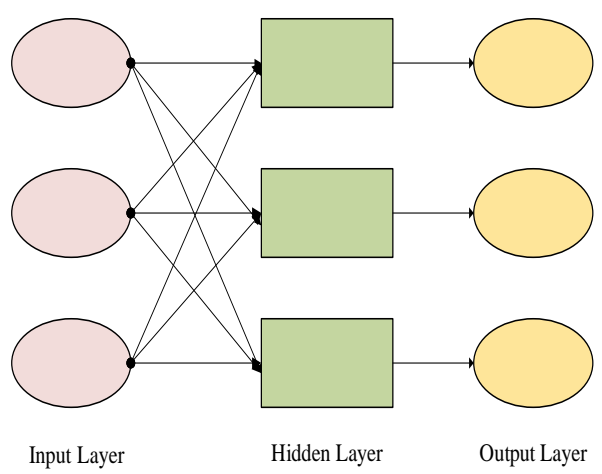

Figure 3: Multilayer feedforward neural network model

The recurrent neural network consists of several neurons connected end to end. When a neuron receives the output signal from the upper neuron, it will also output a signal to the neuron as input, forming a loop, which greatly improves the ability of neural network to learn and process information. Figure 4 is the structural model.

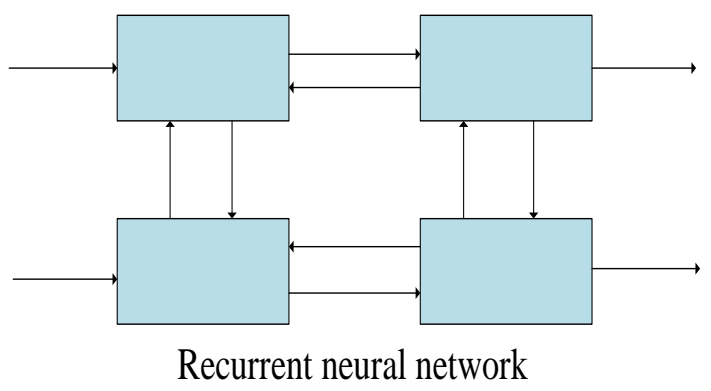

Figure 4: Recurrent neural network model 
As a mathematical model, due to the lack of effective algorithm support, the more the layers of early neural network are, the more complex the model is. There are still many problems in practical application, until Geoffrey Hinton et al. applied the back propagation algorithm to neural network. The neural network trained by back propagation algorithm has gradually formed the most core and widely used BP neural network structure in modern neural network. BP neural network is also called error backpropagation algorithm, because BP neural network is a one-way and forward multilayer neural network, which consists of one input layer, one output layer and several hidden layers; the neurons between two adjacent unit layers correspond to neural nodes one by one; there is no mutual connection between neurons and no influence on each other; the data enters the hidden layer from the input layer, and the output layer outputs the results after calculation. Next, according to the goal of reducing the error between the output direction and the actual target, the weight of input layer to hidden layer is adjusted continuously. This algorithm is called error backpropagation algorithm. With the error being corrected, the network will be more and more accurate in the classification of input data [13]. Figure 5 is its structural model.

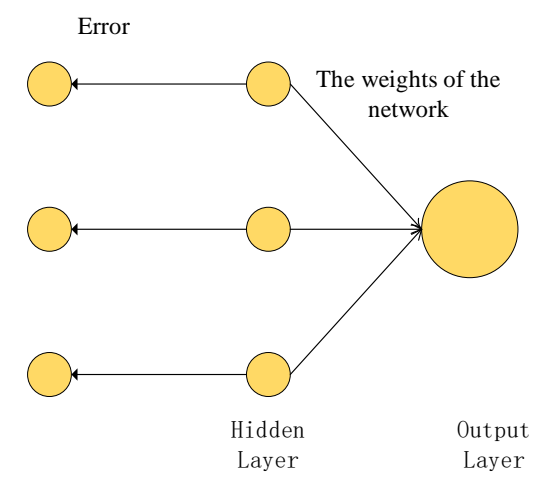

Figure 5: Error backpropagation algorithm model

It shows that neural network is a huge information processing system with high nonlinearity and continuous adaptive. Research shows that under the condition of "universal approximation theorem", it can approach any form of function with any precision. Now, it is widely used in many fields, such as pattern recognition, automatic control, signal processing, auxiliary decision-making, fault detection, and statistics. one of the most potential application fields of artificial neural network is load forecasting of power system [14].

\subsection{The Basic Principle of BP Algorithm}

Step 1: determination of the initial value of node function. The number of nodes $n, l, m$ in the input layer, output layer and hidden layer of the neural network is determined; the connection weight $W_{i j}$ and $W_{j k}$ between the layers, and threshold a, $\mathrm{b}$ for hidden layer and output layer are initialized; then, the error function, training function, error target and maximum learning times are selected.

Step 2: the output data calculation of the hidden layer through equation. Through the hidden layer threshold $\mathrm{a}$, the input vector $\mathrm{X}$ and the network weight $W_{i j}$ between the input layer and the hidden layer, the output $\mathrm{H}$ of the hidden layer is calculated ( $\mathrm{f}$ is the activation function of the hidden layer).

$$
H_{j}=f\left(\sum_{i=1}^{n} W_{i j} x_{i}-a_{j}\right) \quad j=1,2 \ldots l
$$

Step 3: the results calculation of the output layer through equation. Through the hidden layer outputs $\mathrm{H}$, the output layer threshold $\mathrm{b}$, and the connection weight $W_{j k}$ between the hidden layer and the output layer, the output value 0 of the network can be calculated. The calculation equation of 0 is as follows.

$$
O_{k}=\sum_{j=1}^{1} H_{j} w_{j k}-b_{k} \quad k=1,2, \ldots m
$$

Step 4: error calculation. From the expected output $Y$ and the output value $O$ of the network, the prediction error e can be calculated according to the calculation equation.

$$
e_{k}=Y_{k}-O_{k} \quad k=1,2, \ldots m
$$

Step 5: connection weight correction. Through the calculated network prediction error e, the connection weight of the network is modified, and the adjustment method is shown in equations (1-4) and (1-5).

$$
\begin{aligned}
& W_{i j}=w_{i j}+\mathrm{\eta} H_{i}(1-H)_{i} x(i) \sum_{k=1}^{m} w_{j k} e_{k} \\
& i=1,2 \ldots n ; j=1,2, \ldots l \\
& H_{j}=f\left(\sum_{i=1}^{n} w_{i j} x_{i}-a_{j}\right) \\
& j=1,2, \ldots l
\end{aligned}
$$

Step 6: threshold correction. According to the network prediction error e, the thresholds $a$ and $b$ of the hidden layer and the output layer are corrected. The equation is shown in (1-6) and (1-7).

$$
\begin{aligned}
& A_{j}=a_{j}+\mathrm{\eta} H_{j}\left(1-H_{j}\right) \sum_{k=1}^{m} w_{j k} e^{k} \\
& j=1,2, \ldots l \\
& b_{k}=b_{k}+e_{k} \quad k=1,2, \ldots m
\end{aligned}
$$

Step 7: end. The error value and the error threshold are judged. If the requirements are met, the training is over; if not, it is necessary to return to step 2 and continue the training [15]. Figure 6 shows the specific operation process. 


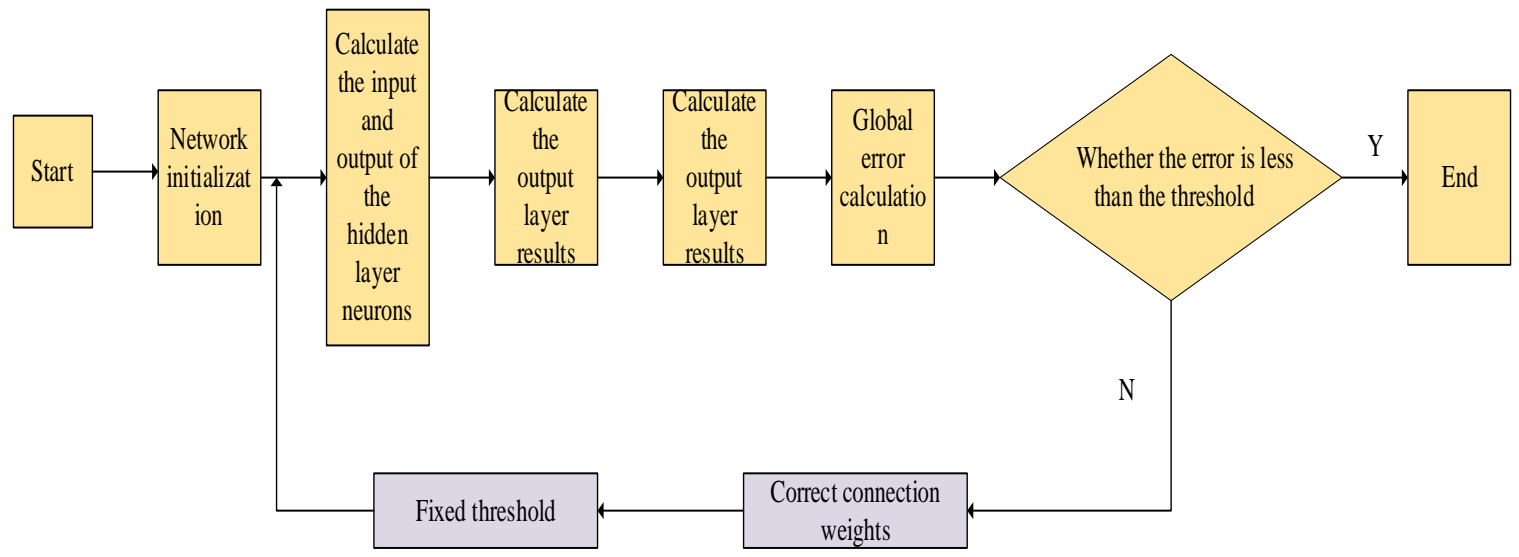

Figure 6: Basic flow chart of BP algorithm

\subsection{Basic Principles of Power Load Forecasting}

Power system short-term load forecasting is mainly to predict the power load value of a certain day or several days in the future, which is a very important work of multiple power management departments in the whole power system. The accuracy of power system load forecasting will directly affect the expansion and capacity increase of power grid. If the normal operation of the power system cannot be guaranteed, it will bring irreversible disasters and economic losses to the power system. If a perfect power system load forecasting system is established, the power system can carry out real-time power dispatching according to the real-time load forecasting information, ensure the stable operation of the whole power system, reduce accidents and reduce economic losses.

Therefore, it is of great significance to study the load forecasting method of power system and develop a power load forecasting analysis system with practical application value [16]. Short term load forecasting is one of the most widely used load forecasting methods in power system. However, some of the traditional load forecasting methods are not suitable for the load forecasting of a certain area, some cannot accurately reflect the trend of the future load, or the steps of load forecasting are very complicated, and they basically cannot be truly computerized. By analyzing the classification and characteristics of power load, some factors affecting load forecasting are summarized. The neural network algorithm is improved by adding influence factor mapping and adding steepness factor to hidden layer function. This method solves the problems of slow convergence speed, and easily falling into paralysis and local minimum in neural network training. Then, the improved neural network algorithm is used to predict the load of an area in Shanghai. When the network load forecasting model is built, the accuracy of network forecasting with meteorological factors will also be improved. When the network is trained, lots of samples covering as many time points as possible are used, and the network weights obtained by training are saved. In this way, the universality of the trained network will be enhanced, and it can be easily used by users in the near future.

\subsection{Design of Power Load Forecasting Framework}

Step 1: determine the forecast direction and make the forecast plan

First, the reasonable and feasible prediction target and content are determined. Then, according to the specific requirements of the forecast content, the specific forecast implementation plan is formulated, and the required relevant information is widely collected.

Step 2: investigate and select data

After a comprehensive analysis of lots of data, the representative data with high degree of authenticity are selected as the basic data. In addition, the abnormal data in the data are analyzed, removed and corrected. One of the basic data for forecasting is historical load data. The historical data is transformed from the original EXCEL form into a text file.

Step 3: obtain the data of power system related factors

Power system is never an isolated system, and it is affected by economic development, national policies, weather changes and other factors. The different data needed are obtained from different departments, and some related factors can be predicted when necessary.

Step 4: select the model and method suitable for the region

According to the determined prediction content, the actual situation of the region and the availability of data are considered, and the appropriate model is selected.

Step 5: establish the prediction model

The concretization of the model is the load forecasting equation. Generally, some mature models should be selected to predict. 
Step 6: preprocess the data

For sample data, on the one hand, it is necessary to eliminate the false data; on the other hand, it is necessary to transform the data according to the different selected models. If the model is BP neural network, it is necessary to normalize the original data; if the model is gray system, it is necessary to "generate" the data; in some models, it is necessary to smooth the historical data.

Step 7: identify model parameter

After the model is established, the parameters of the model can be obtained according to the actual data.

Step 8: evaluate and test the model

According to the hypothesis testing principle, whether the model is suitable or not is determined. If not, it is necessary to discard and replace the model and repeat steps 5 to 8 .

Step 9: apply the model to prediction, and analyze and evaluate the prediction results

According to the selected model and model parameters, the processed sample data are input to make a prediction for the future period, and the prediction results are compared and comprehensively analyzed to determine the pros and cons of the prediction results of different methods or model structures [17].

\subsection{Determination of the Node Function of the Middle Layer}

According to the above design steps, it is necessary to determine the function of the network input and output layer section. When BP neural network is used for prediction, the selection of hidden layer and output layer functions will directly affect the accuracy of output results [18]. According to several groups of functions available at present, the method of testing one by one is generally adopted to set up three groups of tests, and use the same hidden layer function to test different output layer functions. The same network structure, algorithm and input samples are used in the test process, and the same threshold is set for simulation.

\subsection{Data Normalization}

In order to avoid the saturation phenomenon of neurons [19], it is necessary to normalize the input data, and process the data into the data between the interval $[-1,1]$. Meteorological characteristics and other influencing factors are selected from the index mapping database or calculated according to the instructions of the mapping database, which will be conducive to the convergence of the training process. Otherwise, the network may not converge

$$
y=\frac{x-x_{\min }}{x_{\max }-x_{\min }}
$$

$x$ is the value before mapping and $y$ is the value after mapping. After the training, the following equation is used to reverse the normalization.

$$
x=y\left(x_{\max }-x_{\min }\right)+x_{\max }
$$

\subsection{Establish the Mapping Database of Related Influencing Factors}

In fact, there are many factors that need to be considered in power load forecasting, including historical data, week type, meteorological factors, temperature factors and so on. Considering the types of working days, the seven days of a week are divided into working days and rest days according to the composition of the selected samples, that is, Monday to Friday are working days, and Saturday and Sunday are rest days. The mapping values of the two types of days are obviously different. In order to ensure the comparability of various factors, the values taken before the mapping of characteristic quantities corresponding to various factors are mapped to $[0,1]$ interval. The difference between the characteristic quantities is obvious, and the value of more than 1 can be considered. The value before mapping can be defined by itself, but there should be a sort that can be distinguished. For meteorological indexes that can be directly quantified, such as daily maximum temperature, daily minimum temperature, daily average temperature, rainfall, wind speed, and humidity, linear or piecewise linear mapping is considered; for week type, group mapping is used [20].

In order to verify the importance of influencing factors on power load forecasting, two groups of data are tested respectively. In the first group, the meteorological factors and working day types are considered, and the simulation is carried out according to the mapping database method mentioned above. In the other group, only historical data are selected as samples, regardless of meteorological factors and working day types.

Because load curve is a nonlinear function related to many factors, neural network is a very suitable method for extracting and approximating this nonlinear function. The advantage of neural network is that it can simulate multiple variables without making complex assumptions about the input variables. Neural network does not need to rely on expert experience knowledge. It can extract and approximate the implicit nonlinear relationship between input and output through learning in the training process only by using the observed data. There are many researches on short-term load forecasting based on artificial neural network. In recent years, research shows that compared with technical statistics and expert system method, shortterm load forecasting using neural network technology can obtain more accurate forecasting data [21]. 


\subsection{Selection of Sample Data}

The historical load data of a certain area is selected. If every day of the week is taken as a type, there are seven types in a week. Every two hours, the power load of the day before the forecast day is measured, so that a total of 12 groups of load data can be measured in one day.

Through long-term monitoring, there is no mutation between the adjacent points of the load curve, so the value of the next moment is related to the value of the previous group.

The load data of the previous day can be taken as the network sample data without considering the occurrence of major accidents and other special circumstances. The daily load data from July 7, 2020 to July 17, 2020 and the weather characteristics and week types of the next day in Songjiang District of Shanghai are selected as the input vectors. A total of 11 groups of data are shown in Tables 1 and 2 .

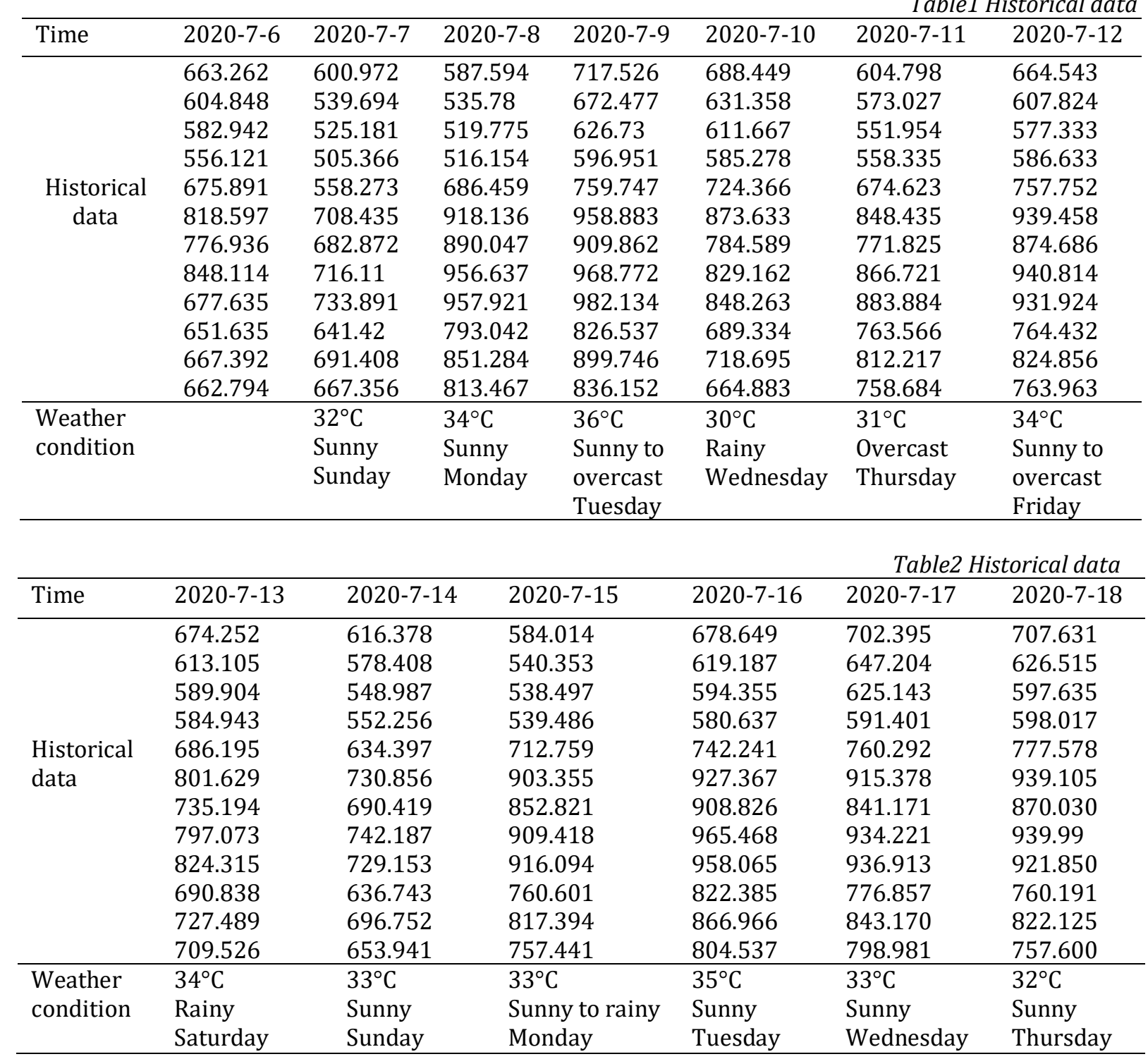

In the experiment, the daily load data from July 8 , 2020 to July 18, 2020 will be used as output vectors, and a total of 11 groups of vectors will be trained and learned; the load data from July 17, 2020 and the weather characteristics and week types on July 18, 2020 will be used as test samples to predict the load on July 18, 2020 for test. It can be put into use only after passing the inspection. The corresponding neural network structure is a three-layer network structure. In order to optimize the network structure, the selection of the number of neurons in the middle layer is discussed through simulation and comparison. According to the above network model, from the sample data, it can be known that there are 16 neurons in the input layer of the network.

According to Kolmog-orow theorem, there are 33 neurons in the middle layer of the network. However, in order to improve the accuracy of prediction, in the experiment, 23, 28, 33, 40 neurons will be selected as four groups of experiments for comparison, and trained and simulated respectively.

The results are compared to choose one of the 
best scheme. The transfer functions of the middle layer and the output layer are S-shaped. According to the verification of the convergence speed of all algorithms at present, the improved algorithm based on Levenberg-Marquardt method has faster convergence speed. In the training, it is adopted, namely L-M algorithm [22].

Before the simulation, the established BP neural network model should be trained for 1000 times. The training goal is set as 0.01 and the learning rate is 0.1. Based on the trained neural network, simulation can be started. According to the error between the predicted value and the real value, the rationality and feasibility of the experiment are tested.

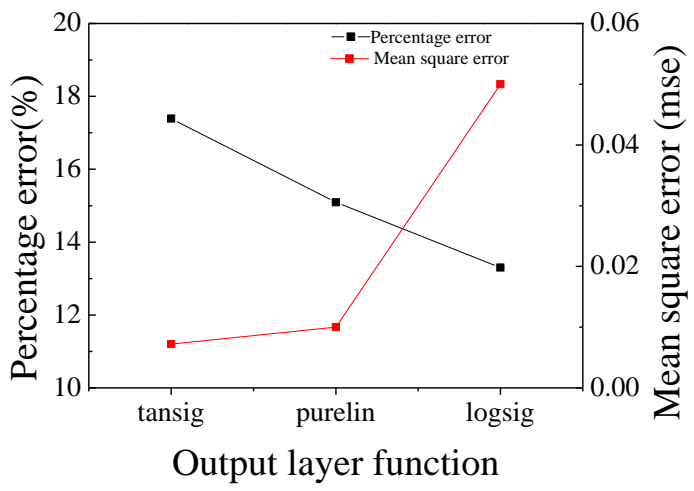

(a)
In the experiment, Out is the predicted value, and Error is the difference between the predicted value and the real value.

\section{Simulation Results of BP Neural Network 3.1 The Influence of Middle Layer Function}

According to the choice of different input layer and hidden layer functions, Figure 7 shows the error percentage and mean square error between the output result and the real value respectively.

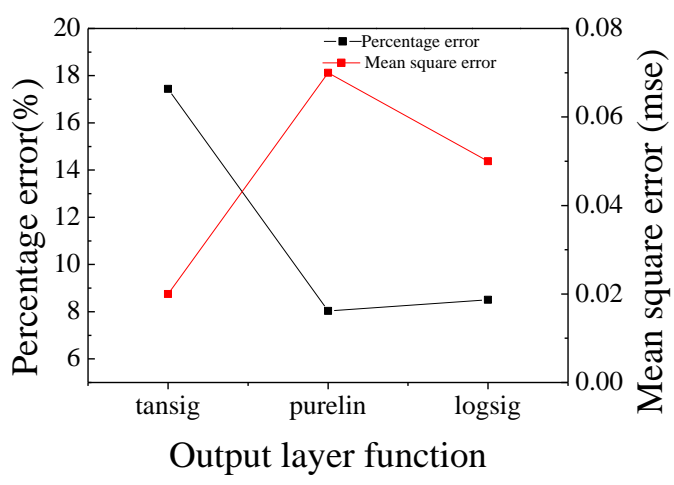

(b)

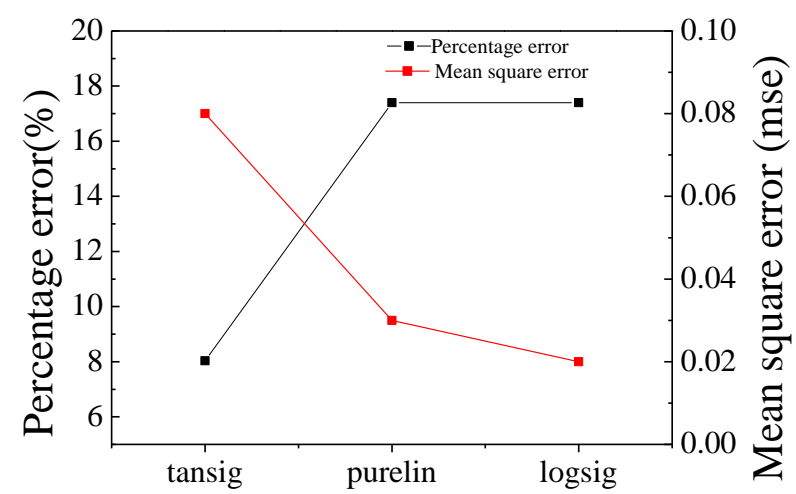

Output layer function

(c)

Figure 7: Error percentage and mean square error of different hidden layer functions

(a). logsig; (b). tansig; (c). purelin

It reveals that the different choice of node transfer function between hidden layer and output layer will also have a greater impact on the prediction accuracy. According to the error statistical analysis of different hidden layer and output layer functions, it is decided that tansig function or logsin function should be selected for node transfer function of hidden layer, and purelin function or tansig function should be selected for output layer transfer function.

\subsection{The Influence of the Number of Neurons in the Middle Layer}

According to the L-M algorithm, when the middle layer neurons (expressed as n) are 23, 28, 33 and 40, respectively, Figure 8 shows the error between the prediction result and the real value generated by BP neural network. 


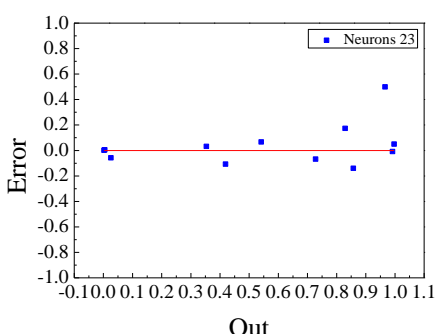

(a)

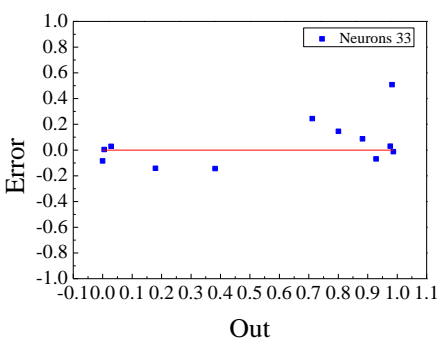

(c)

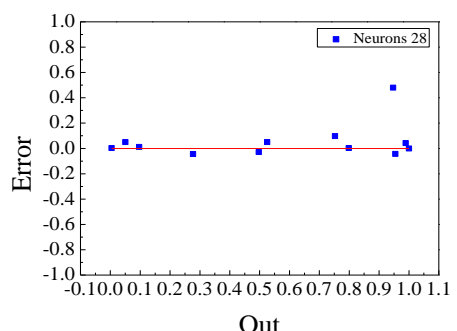

(b)

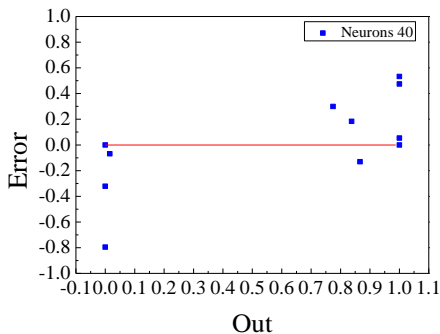

(d)

Figure 8: The error of the prediction result with different number of middle layer neurons (a). 23 neurons in the middle layer; (b). 28 neurons in the middle layer; (c). 33 neurons in the middle layer; (d). 40 neurons in the middle layer

Figure 8 suggests that when the number of neurons in the middle layer is 28 , the error is small; when the number of neurons in the middle layer is 33 , the network convergence speed is faster, but the error is larger than that when the number of neurons in the middle layer is 28 . It also proves such an important conclusion. Although the number of neurons in the middle hidden layer is positively correlated with the accuracy of neural network mapping, there is the problem of over fitting. In the experiment, faster convergence does not mean better results, and the performance of neural network needs to be considered. Therefore, when the number of neurons in the middle layer is selected, it is necessary to select the one with relatively small error instead of blindly pursuing the number.

\subsection{The Important Role of Impact Factor Mapping Database}

In order to verify the importance of influencing factors on power load forecasting, two groups of data are tested respectively. In a set of data, the meteorological factors and working day types are considered, and the simulation is carried out according to the mapping database method mentioned above. In the other group, only historical data are selected as samples, regardless of meteorological factors and working day types.

In the first group of experiments, the influence factors are considered, and the mapping library needs to be established according to the perturbation algorithm described in the previous section. Figure 9 shows the specific values.

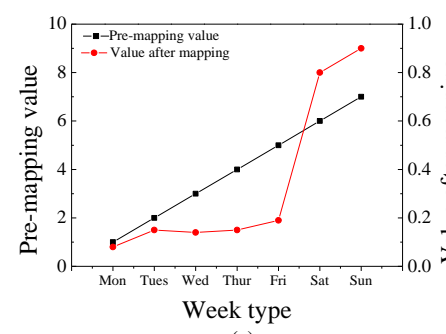

(a)

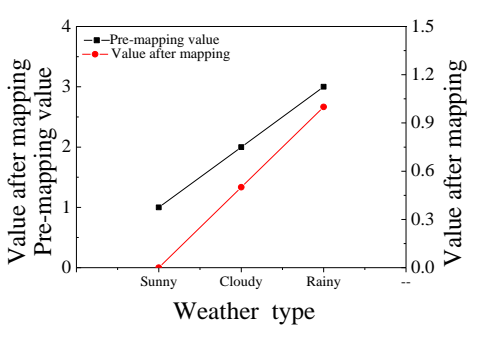

(b)



(c)

Figure 9: The values of different influence factors before and after artificial mapping

(a) week type; (b) weather type; (c) temperature type 
For the index mapping data in Figure 9, because the working days type always plays a dominant role in the short-term load influencing factors, it is mapped between [0,1]. The mapping values from Monday to Friday are relatively close, while the rest days, Saturday and Sunday, are quite different from working days.

Therefore, the mapping values of working days and rest days are set to 0.8 and 0.9 to show the difference between them.

For meteorological factors, with the maximum temperature as an example, the relationship between the local maximum temperature and load is analyzed. $30^{\circ} \mathrm{C}$ and $35^{\circ} \mathrm{C}$ are taken as the maximum temperature threshold, linear mapping is used between $0^{\circ} \mathrm{C}$ and $30^{\circ} \mathrm{C}$, and the mapping value changes little; another group of linear mapping is used between $30^{\circ} \mathrm{C}$ and $35^{\circ} \mathrm{C}$; when the temperature is above $35^{\circ} \mathrm{C}$, the load will increase greatly with the increase of $1^{\circ} \mathrm{C}$, so the nonlinear mapping is used to show the difference.

For the second group of data, the same BP network model and improved algorithm of the first group will be used. There are 12 samples in each group, 12 neurons in the input layer, the number of training is still set at 1000 , the training goal is 0.01 , and the learning rate is 0.1 . Figure 10 shows the error between the predicted results and the real values.



(a)

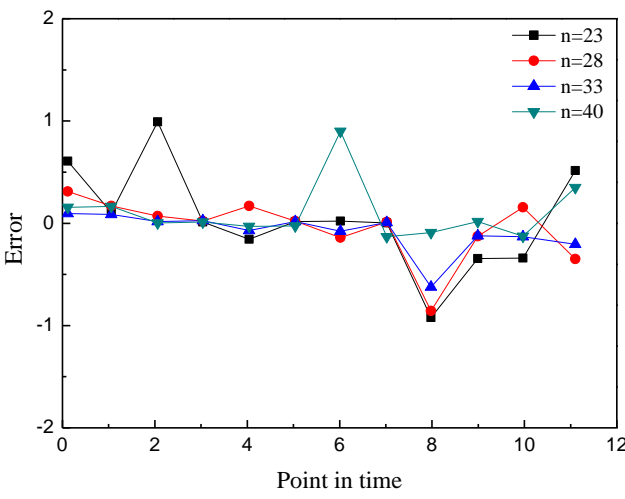

(b)

Figure 10: The error between the predicted result and the real value (a). considering meteorological and working day factors; (b). without considering meteorological and working day factors

Figure 10 reveals that the prediction result without considering the daily meteorological characteristics and week type has larger error than that considering the daily meteorological characteristics and week type. It is obvious that the prediction effect with considering the daily meteorological characteristics and week type is better. The forecast without considering the daily meteorological characteristics and week type only needs the historical load data, and the sample data is few, so the training steps are less under the condition of using the same algorithm. The simulation results without considering the influence factors (weather and time characteristics) are compared with that of considering the influence factors, and the following conclusions can be obtained. The establishment of mapping database is conducive to the prediction accuracy improvement of neural network. The more the influence factors are considered, the more perfect the mapping function library is, and the higher the accuracy of load forecasting using neural network is.

\section{Conclusion}

The concept of BP neural network and its practical application in electrical automation system are mainly introduced. By establishing the mapping database of the actual factors affecting the power load, and using the BP neural network based on L-M algorithm, the specific prediction scheme in line with the actual situation is designed. According to the simulation results, compared with the traditional expert prediction method,

BP neural network trained by genetic algorithm can get smaller error between the predicted results and the actual results, and the average error can be controlled within 0.05 (when the hidden layer function is tansig function or logsin function, the output layer transfer function is purelin function or tansig function, and the number of nodes in the middle layer is set to 28). Meanwhile, after the influence of meteorological factors and working day factors is considered, it is important to establish a perfect mapping library of influence factors for the optimization of the prediction results, and the effectiveness and stability of the prediction results have been further improved.

The shortcomings of this exploration are as follows. First, the selected historical data does not have significant holiday time nodes. In the establishment of impact factor mapping database, major holidays are not considered. In fact, the impact of major holidays on power load is relatively large. 
Second, there is no in-depth study of BP neural network optimization algorithm, and genetic algorithm itself has defects. At present, there are particle swarm optimization algorithm, batch gradient descent algorithm and so on. The optimization results of different algorithms need to be further studied.

In the near future, the application of improved BP network model for power load forecasting will have higher accuracy. With the development of science and technology, more advanced algorithm and influence factor mapping library will make the function of BP neural network more powerful, which can be effectively put into the operation and maintenance of the actual power grid.

\section{References}

[1] Li B. (2020) Artificial Intelligence Technology in Electrical Automation Control. Home Science and Technology, 335(11),22-23.

[2] Yu H., Zhang Z., Gomg W, et al. (2019) Short-term Load Prediction Model of Power System Based on Deep Recursive Neural Network. Journal of Power Systems and Automation, 31(1), 112-116.

[3] Zhang B. (2017) Fault Diagnosis of Power Electronic Circuit Based on DSP Artificial Neural Network. Electronic Measurement, 382(24), 1414.

[4] Hu Y., Liu C., Chen Y, et al. (2019) Neural Network Power Load Prediction Based on Principal Component Analysis. Automation Technology and Application, 38(7), 90-93.

[5] Wang C., Huang Q., Cai X, (2020) Fault Diagnosis of Flexible Manufacturing System Based on BP Neural Network Algorithm. Tool Technology, 561(5), 74-77.

[6] Yuan X., Li G., Xu A, et al. (2020) Short-term Power Load Prediction Based on Improved and Enhanced Neural Network. Computer and Digital Engineering, 364(2),227-232+237.

[7]Jiang X, Guo Y, Shi H, et al. (2019) Predictive Control Based on TSA Multivariable Nonlinear RBF Neural Network. Control Engineering, 177(9), 53-58.

[8] Wang J. (2019) Fault Detection Method of Electrical Automation Equipment Based on Neural Network. Automation \& Instrumentation, 242(12), 155-158+163.

[9] $\mathrm{Hu}$ Y., Liu C., Chen Y, et al. (2019) Neural Network Power Load Prediction Based on Principal Component Analysis. Automation Technology and Application, 38(7),90-93.

[10] Gurney K. (2019) Neural networks for perceptual processing: from simulation tools to theories. Philosophical transactions of the Royal Society of London. Series B, Biological sciences, 1479, 339-53.
[11] Xiang D., Wang B., Guo W, et al. (2017) Refined Safe Operation Rules of Power System Based on Artificial Neural Network. Power System Protection and Control, 56(18), 32-37

[12] Luo K., Zhang Y. (2019) Research and Application of Energy Management System Based on BP Neural Network Prediction Model. Industrial Control Computer, 32(9),50-51+53.

[13] Li C., Guo Wenli., Wu Jin, et al. (2019) Prediction Method of Daily Maximum Power Load in Beijing Summer Based on BP Neural Network. Climate and Environmental Research, 24(1), 135-142.

[14] Li G., Liu Z., Jin G, et al. (2020) Ultra-short-term Power Load Prediction Based on Random Distributed Embedding Framework and BP Neural Network. Power Grid Technology, 435(2), 53-61.

[15] Venkatanagaraju K., Biswal M., Abdelaziz A Y. (2021) Protection system failure and power system blackout ScienceDirect. Uncertainties in Modern Power Systems, 7,257-296.

[16] Li W. (2017) Research on Power Load Prediction Model Based on Elman Neural Network Algorithm. Journal of Chifeng University (Natural Science Edition), (21), 43-45.

[17] Gao X. (2017) Application of Electrical Automation in Power Supply and Distribution System. Doors and Windows, 7, 245-245.

[18] Yaqoob M T., Alam M., Rahmat M K, et al. (2020) A Review on Importance of Active Learning Neural Network for Grid Connected Renewable Energy System. Journal of Computational and Theoretical Nanoscience, 17(2), 629-634.

[19] Zhang Z., Zhao J., Lu X, et al. (2017) Application of BP Neural Network Based on Genetic Algorithm in Power Load Prediction. Computer Engineering, 10, 277-282.

[20] Huang Q., Wang H. (2020) Wind power forecast based on convolutional neural network with multi-feature fusion. Journal of Physics: Conference Series, 1684(1), 12134.

[21] Pandian S. (2020) Comparative Analysis of Sliding Mode Control, Artificial Neural Network and Fuzzy Logic controlled based IDVR for Power System Stability. The International journal of analytical and experimental modal analysis, 29(7), 11208-11218.

[22] Nureddin A A M., Rahebi J., Ab-Belkhair A. (2020) Power Management Controller for Microgrid Integration of Hybrid PV/Fuel Cell System Based on Artificial Deep Neural Network. International Journal of Photoenergy, 1, 21. 